Published in final edited form as:

Nat Genet. 2017 April ; 49(4): 497-503. doi:10.1038/ng.3800.

\title{
Accounting for genetic interactions improves modeling of individual quantitative trait phenotypes in yeast
}

\author{
Simon K. G. Forsberg ${ }^{1}$, Joshua S. Bloom ${ }^{2,3,4}$, Meru J. Sadhu ${ }^{2,3,4}$, Leonid Kruglyak ${ }^{2,3,4}$, and \\ Örjan Carlborg ${ }^{1,}$ \\ ${ }^{1}$ Department of Medical Biochemistry and Microbiology, Uppsala University, Uppsala, Sweden \\ ${ }^{2}$ Department of Human Genetics, University of California, Los Angeles, Los Angeles, California \\ 90095, USA \\ ${ }^{3}$ Howard Hughes Medical Institute, University of California, Los Angeles, Los Angeles, California \\ 90095, USA \\ ${ }^{4}$ Department of Biological Chemistry, University of California, Los Angeles, California 90095, USA
}

\begin{abstract}
Experiments in model organisms report abundant genetic interactions underlying biologically important traits, whereas quantitative genetics theory predicts, and data support, that most genetic variance in populations is additive. Here we describe networks of capacitating genetic interactions that contribute to quantitative trait variation in a large yeast intercross population. The additive variance explained by individual loci in a network is highly dependent on the allele frequencies of the interacting loci. Modeling of phenotypes for multi-locus genotype classes in the epistatic networks is often improved by accounting for the interactions. We discuss the implications of these results for attempts to dissect genetic architectures and to predict individual phenotypes and long-term responses to selection.
\end{abstract}

\section{Introduction}

When the combined phenotypic effect of alleles at two or more loci deviates from the sum of their individual effects, it is referred to as a genetic interaction or epistasis. Most biological traits are regulated by a complex interplay between multiple genes and environmental factors. Despite this underlying complexity, data and theory have shown that it is expected that most of the genetic variance in a population will be additive ${ }^{1-3}$. The apparent

Users may view, print, copy, and download text and data-mine the content in such documents, for the purposes of academic research, subject always to the full Conditions of use: http://www.nature.com/authors/editorial_policies/license.html\#terms

*Correspondence should be addressed to Ö.C. (orjan.carlborg@imbim.uu.se).

Data Availability Statement

The genotype and phenotype data that was re-analyzed in this study are available in the Supplementary information of the original publication for which this data was generated ${ }^{3}$.

Author Contributions

Analyses were designed by S.K.G.F., J.S.B., M.J.S., L.K. and Ö.C. Analyses were conducted by S.K.G.F and Ö.C. The manuscript was written by S.K.G.F. and Ö.C. and incorporates comments by J.S.B., M.J.S. and L.K.

Competing Financial Interests Statement

The authors declare that they have no competing financial interests. 
contradiction between the complexity of the biological mechanisms that determine quantitative traits, and the observation that most genetic variance can be captured by an additive model, has led to a long-standing debate in genetics: Does the predominant role of the additive genetic variance mean that strictly additive models are always sufficient to describe the relationship between the genotype and the phenotype of an organism ${ }^{1,4}$, or could there be added value in explicitly modeling genetic interactions despite the lower levels of epistatic genetic variance ${ }^{5-8}$ ?

There are some situations where data and theory has suggested that it might be particularly important to account for genetic interactions. One is when the aim is to predict phenotypes of individuals based on their genotype. If interactions lead to extreme phenotypes for some genotypes, these phenotypes are unlikely to be captured by additive models, particularly if they are rare. This has, for example, been illustrated for sporulation efficiency in yeast ${ }^{9}$. Another is in the prediction of long-term selection response. Under additivity, both the additive variance and the response are expected to be near constant over the first few generations. As generations proceed, allele frequencies change to alter the additive variance and consequently the response to selection. This change is more rapid for traits regulated by fewer loci with larger effects than for traits regulated by many loci with smaller effects. It is known that genetic interactions can contribute to the additive genetic variance in a population ${ }^{1,7}$. The contribution, however, varies depending on the joint allele frequencies across all the interacting loci as well as on the types and strengths of the genetic interactions ${ }^{10,11}$. The changes in the additive variance, and hence the response, during ongoing selection is therefore more complex in the presence of genetic interactions. As a result, genetic interactions can make the long-term selection response more dynamic ${ }^{12,13}$ and result in a realized response beyond predictions based on the additive genetic effects and allele frequencies at the individual loci ${ }^{10,11}$. However, as little is known about how prevalent and strong genetic interactions are in real populations, and how much they contribute to the additive variance as the allele frequencies change during selection, it has been difficult to obtain any empirically based conclusions about how influential interactions are expected to be in these situations.

Here we analyze a panel of 4,390 yeast recombinant offspring (segregants) from a cross between a lab strain (BY) and a vineyard strain (RM), generated in Bloom, et al. $2015^{3}$. In this population, each segregant is genotyped for 28,220 SNPs and phenotyped for 20 endpoint growth traits. Across these traits, a total of 939 QTL with additive, and 330 with epistatic, effects were mapped previously ${ }^{3}$. Since the individuals in this population are haploids, the sample size is large and all allele frequencies are close to 50\%, this dataset presents a unique opportunity to accurately estimate how allelic combinations across large numbers of loci influence quantitative traits. Using phenotype information for multiple segregants with each possible allelic combination across many loci, we directly estimate how high-order genetic interactions contribute to complex trait variation in this segregating population. We quantify how well quantitative genetics models can capture the empirically revealed relationships between multi-locus genotypes and phenotypes.

We observe networks of epistatic loci for most of the traits in the study, and find that some highly interactive loci in these networks can hide, or reveal, the effects of their interactors. 
We show that additive genetic models capture much of the genetic variance contributed by the interacting genes in these networks. However, when used to estimate the phenotypes for individual segregants, they often fail to fully capture the effects of multi-locus genotypes that lead to extreme phenotypes. Accounting for interactions in the models led to more accurate phenotypic predictions for such genotypes. We illustrate this by analyzing an individual network in detail, and then generalize the results across the other revealed networks. We discuss the potential impact of these findings on prediction of individual phenotypes that is of importance in for example personalized medicine, prediction of longterm selection response in breeding and evolution, and interpretation of results from QTL and genome-wide association studies.

\section{Results}

\section{Many epistatic QTL are part of highly connected networks}

Most epistatic QTL ${ }^{3}$ interacted with one or a few loci, while a smaller subset were involved in pairwise interactions with several loci (Figure 1a). By visualizing the 330 statistically significant epistatic QTL $^{3}$ as nodes, and the interactions between them as edges, we revealed many networks of interacting loci. These were often connected in hub-and-spoke type of architectures where QTL involved in many interactions tied larger networks together (Figure $1 b)$. We refer to these as radial networks, with a hub-QTL in the center that connects the radial QTLs. Hub-radial QTL interactions were, on average, more significant than interactions that did not involve a hub (Supplementary Figure 1), supporting that the radial architecture is a prominent feature of the networks. The available genotype and phenotype data allowed us to accurately estimate the phenotypes for individual six-locus genotypeclasses (see below). We therefore selected the 15 six-locus radial networks where a hubQTL interacted with at least five other QTL, for further in-depth studies (Figure 1b). The selected networks contributed to 11 of the 20 studied traits, and included 81 QTL.

Below, we first illustrate our analyses and results for the network regulating growth on medium containing indoleacetic acid (IAA-network) and then extend them to all networks.

\section{Hub-QTL often moderate the phenotypic effects of radial QTL}

The hub-QTL in the IAA-network explained the most additive genetic variance of any locus for this trait $\left(\frac{\sigma_{a}^{2}}{\sigma_{p}^{2}}=11 \%\right) 3$. The phenotypic variance was 3-fold higher among segregants with the BY-genotype, than among segregants with the RM-genotype, at this locus. This genetic variance-heterogeneity is highly significant ( $p<2.2 \times 10^{-16}$; dglm, two-sided test). By estimating the narrow sense heritability $\left(\mathrm{h}^{2}\right)$ separately among segregants with the BY and RM allele at this hub-QTL we showed that much of this difference was due to genetics ( $h_{B Y}^{2}=0.55 \mathrm{vs} h_{R M}^{2}=0.14$ ). We here call such QTL, where one allele suppresses genetic contributions by other loci and the other allele uncovers them, genetic capacitors. Across the 15 epistatic networks, 10 hub-QTL were genetic capacitors with significant differences in $\mathrm{h}^{2}$ ( $p<0.001$; Bonferroni corrected multiple-testing threshold; one-sided permutation test) between the genotypes ranging from 10 to $42 \%$. By testing 40 randomly selected radial QTL, we found that few (3) of these were significant genetic capacitors and that they were 
weaker capacitors than the hubs (mean difference \pm SD in $h^{2}$ for radial QTL $=4.3 \% \pm 3.6$ vs $12.5 \% \pm 9.2 \%$ for hub-QTL). We also revealed a strong correlation $(r=0.64$; Pearson correlation; $p<2.2 \times 10^{-16}$; two-sided test) between the level of variance-heterogeneity between the genotypes at the 330 epistatic QTL and the number of interactions they were involved in (Supplementary Figure 2). Together, this shows that strong genetic capacitors $^{14,15}$ often are hubs in epistatic networks.

\section{Modeling phenotypes for multi-locus genotype-classes}

Allelic interactions contribute to complex trait variation-For the six-locus IAAnetwork, we divided the segregants into 64 groups representing each of the 64 possible sixlocus genotype classes, and calculated their phenotypic means and variances (Figure 2a). Segregants with the capacitating BY allele at the hub-QTL (green) display the poorest growth when they have many IAA-sensitizing alleles at the five radial QTL. In contrast, segregants with the canalizing RM allele at the hub-QTL (grey) have similar growth regardless of how many IAA-sensitizing alleles they have at the radial QTL. The RM/BY alleles at the hub-QTL in the network thus decrease (canalize)/increase (capacitate) the effects of the radial QTL, respectively (Figure 2a). Similar results are also observed for several of the other networks with significant differences in $\mathrm{h}^{2}$ between the genotypes at the hub-QTL ( $p<0.001$; Bonferroni corrected multiple-testing threshold; one-sided permutation test; Supplementary Figure 3).

Across the networks, we detected hub-QTL capacitor alleles of both BY and RM origin. The most extreme phenotypes in these networks were always observed for a genotype-class with a combination of BY and RM alleles at the hub and radial loci. The alleles at the radial loci that required the presence of the capacitating hub allele to reveal their full effect on growth (Figure 3; Supplementary Figure 3; Supplementary Figure 4) did in 60\% of the cases originate from the same strain as the canalizing hub allele. The two parental strains thus harbor cryptic, or hidden, genetic variation ${ }^{16-19}$, whose phenotypic effect is revealed when combined in the haploid segregants.

Non-linear capacitation effects in some epistatic networks-In the IAA-network, the reduction in growth among the segregants with the BY allele at the hub-QTL decelerates in a multiplicative, rather than a linear, manner as the number of IAA-sensitizing alleles increases (Figure 2b). For segregants with the BY allele at the hub-QTL, the effect of having 5 IAA-sensitizing alleles is much larger than 5 times the effect of having one IAAsensitizing allele. As a result, an exponential model fits this data better than an additive model (R2 increases from 0.34 to 0.39; Figure $2 \mathrm{~b}$ ). One other network, affecting growth in medium containing Copper Sulfate, displayed a similar non-linear capacitation (R2 increased from 34 to 43\%; Figure 3d; Supplementary Figure 4). This multiplicative effect could result from measuring growth as the increase in radius of the yeast colonies, or it could be a feature of the underlying biology.

Extreme estimates from additive models are often biased-Many additive-model based estimates of the phenotypes for multi-locus genotype classes in the IAA network differed substantially from the actual values estimated directly from the data (Figure 2a). 
Cross-validated model-based estimates were computed for each multi-locus genotype class to quantify their accuracy and bias. Accuracy for each genotype class was measured by the mean square error (MSE), and bias by the difference between the modeled and actual phenotypes. Twenty-three of the 64 estimates were significantly biased (Bonferroni corrected multiple-testing threshold ( $p<\frac{0.05}{64}$ ); two-sided t-test), showing that the additive model was unable to represent the genetic contributions by the IAA network to many of the individual segregant phenotypes. To evaluate whether this trend generalized across all networks, and whether alternative quantitative genetics model parameterizations could perform better, we fitted three different quantitative genetics models to all six-locus networks: i) additive effects only, ii) additive effects and pairwise interactions and iii) additive, pairwise and three-way interactions.

Models with only additive effects captured much of the phenotypic variance for all networks (on average $28 \%$ of $\sigma_{p}^{2}$ ). Accounting for epistatic interactions only increased the variance explained marginally (on average 5\% of $\sigma_{p}^{2}$ ). The additive model based estimates of the phenotypes were, however, significantly biased for between one and 23 of the 64 genotype classes per network in 14 of the 15 examined networks (Bonferroni corrected multipletesting threshold ( $p<\frac{0.05}{64}$ ) two-sided t-test; Figure 2; Figure 4; Supplementary Figure 5). Models with pairwise interaction terms provided unbiased estimates of all 64 measured genotype values for most networks. Only two networks required three-way interaction terms to remove all detectable bias. In 5 of the 15 networks, the accuracy was significantly better for at least one of the 64 genotype classes when using models with pairwise interaction terms (Bonferroni corrected multiple-testing threshold ( $p<\frac{0.05}{64}$ ); two-sided t-test; Supplementary Figure 6).

The bias for the additive model estimates of the phenotypes of individual multi-locus genotypes was largest for the most extreme trait values, i.e. those corresponding to the best or worst growth in the capacitated group, and the best or worst estimated growth in the canalized group (Figure 2; Figure 4). In networks where the hub-QTL are capacitors, the direction of the bias depends on the genotype at the capacitor: Among segregants with the capacitor allele at the hub-QTL, the models underestimated the phenotypic effect of combining many growth-increasing or growth-decreasing alleles at the radial-QTL (Figure 4a). For segregants with the canalizing allele at the hub-QTL, the models instead overestimated these phenotypic effects (Figure 4b). By accounting for epistatic interactions, the bias is reduced or entirely removed (Figure 4). For the 5 networks where the hub-QTL was not a significant capacitor, the bias was not dependent on the genotype at the hub-QTL.

\section{Genetic variances explained by loci in epistatic networks}

The additive genetic variance contributed by a locus depends on its effect and allele frequency in the analyzed population ${ }^{20}$. When loci interact, the variance explained marginally by each of the epistatic loci will in addition also depend on the frequencies at the loci with which it interacts. For example, the additive genetic variance contributed by each individual locus in the IAA-network varies depending on the allele frequencies of all loci in the network due to the extensive interactions among them. When considering the entire 
population of segregants (all allele frequencies $\sim 0.5$ ), the additive variance $\left(\sigma_{a}^{2}\right)$ contributed by the network amounts to $26.8 \%$ of the total phenotypic variance in the population. In the subpopulations where the BY/RM alleles at the hub-QTL are fixed (BY allele frequency 1 or 0 ), the network instead contributes $36 \%$ and $3 \%$ of the total phenotypic variance, respectively. To generalize this result across the allele-frequency space, we simulated populations with allele frequencies ranging from 0.05 to 0.95 at increments of 0.15 for the six loci in the network. We then evaluated how the additive genetic variance contributed by the individual loci varied depending on the allele frequencies at the other five loci. We also simulated populations without genetic interactions. In Figure 5 we summarize the results for the simulations based on the 64 actual genotype-values in the IAA-network. The additive genetic variance contributed by the hub-QTL varied from 0 to $58 \%$ of the total phenotypic variance in the population, only by changing the allele-frequencies at the five other loci (Figure 5). The result was similar for the other five QTL, although their ranges were smaller than for the hub-QTL (Figure 5 blue boxes; average range: 0 to $31 \%$ of $\sigma_{p}^{2}$ ). As expected, the additive genetic variance contributed by each locus was much less dependent on the allele frequencies when loci with the same marginal effects, but no interactions, were simulated

(Figure 5 red boxes; range: 2 to $6 \%$ of $\sigma_{p}^{2}$ ). The results were similar across all 15 networks, with estimates of additive genetic variance for individual epistatic loci that were highly dependent on the allele frequencies at the other loci in the network.

\section{Discussion}

The link between the genotype and the phenotype of an organism is immensely complex. Despite this it can, to a great extent, be captured using models that assume that gene variants combine their effects in an additive manner. We here used a large experimental yeast cross to identify six-locus epistatic networks affecting 11 complex traits. We then estimated the average phenotypes for the groups of segregants sharing each combination of alleles at these loci. We evaluated how well different quantitative genetics models captured the phenotypes of these multi-locus genotype classes. In most networks, the phenotypes for at least one, but often several, multi-locus genotype classes deviated significantly from what is expected under additivity. This empirically illustrates the important role of classic epistasis, as defined by Bateson more than 100 years ago ${ }^{21}$, in the genetic architecture of complex traits. We provide several examples of such epistasis, involving multiple loci in highly interconnected genetic networks.

An earlier study of this population ${ }^{3}$ showed that most of the genetic variance for the 20 measured traits is additive. Consistent with this, the additive model based estimates of the phenotypes for most multi-locus genotype classes in the epistatic networks were reasonable. However, the most extreme estimates from the additive models were often both inaccurate and biased (Figure 4). For example, the bias is very large for the most extreme genotype class in the IAA-network (1.7 $\sigma_{p}$; Figure 2). Our results highlight the importance of analyzing collected data with models that can represent the features of the underlying genetic architectures. They also confirm that, regardless of the underlying genetic architecture, additive models are likely to capture much of the genetic variation for a trait. This makes them useful for revealing genes contributing to the phenotypic variance in a 
particular population, as well as for predicting short-term response to selection in a population ${ }^{11}$. However, we also show that additive models are often unable to represent all key features of genetic architectures involving networks of epistatic loci. In particular, their most extreme estimates are often inaccurate and biased for networks with capacitor hubQTL. Accounting for epistasis increases estimation accuracy and decreases bias. Modeling genetic interactions should therefore be considered when it is important to identify and predict the effects of specific combinations of alleles, or where it is important to identify genotypes that are likely to lead to extreme phenotypes. Examples of this include prediction of disease risk or drug responses in individual patients.

As shown here and in earlier studies ${ }^{1,7,22}$, most genetic variance in a population is expected to be additive even in the presence of extensive epistasis. The lack of empirical knowledge about the pervasiveness and strength of epistasis in the genetic architectures of complex traits makes it largely unknown how much of the observed additive genetic variance in quantitative genetics studies is due to genetic interactions. This experimental yeast population allowed us to directly estimate the phenotypes for individual multi-locus genotype classes in networks of interacting loci. With these as a basis, we used simulations to demonstrate that the types of interactions revealed here have a very large influence on both the estimates of the additive genetic effects of the individual loci and their contributions to the additive genetic variance. The IAA-network provides a striking example: the additive variance contributed by the hub in the epistatic network (Figure 2) ranged from zero to the largest contribution by any single locus across all networks when we varied the allele frequencies at the 5 radial QTL. This empirically illustrates how allelic interactions (epistasis) can be the main driver of the additive genetic variance in a population, and that the importance of epistasis in the genetic architecture of a complex trait cannot be inferred from the relative levels of additive and epistatic genetic variance.

Many interacting loci in this population were part of radial epistatic networks where hub loci interact with multiple other QTL. In general, this network topology reflected how the loci contributed to the phenotypic variation in the population. The hub-QTLs acted as genetic capacitors that modify the effects of the radial loci in the network. These capacitating interactions are highly influential for the total level of phenotypic variance displayed in a population, as they can both buffer and release cryptic (standing) genetic variation ${ }^{23,24}$. Several genetic capacitors have been studied in molecular detail, including the heat shock protein $H S P 90^{16,25}$ and $E G F R^{26}$. Genetic capacitation has also been found for complex traits in segregating populations. For example, it facilitated extreme selection responses for body-weight in a long-term experimental selection experiment in chicken ${ }^{10-12}$ and contributed to the variation in root length between natural Arabidopsis thaliana accessions ${ }^{27}$. This study suggests that capacitor networks are likely to be a common property of the genetic architecture of complex traits. We found them to be both common and influential for many traits in this population, suggesting that they should be considered in other studies of complex traits, including those aiming to genetically dissect, or statistically predict, responses to long-term selection.

It is currently unknown how indoleacetic acid affects yeast fitness, but the discovery of the epistatic network described here may shed some light on its mode of action. The hub-QTL 
in the IAA-network maps to the gene GPA1, which is required for the yeast response to mating pheromone. Although this response is not normally triggered under laboratory growth conditions, as the yeast is not exposed to mating pheromone, the BY allele of GPA1 leads to residual expression of the pheromone response pathway ${ }^{28,29}$. Thus, a model for the capacitance activity of the BY allele of GPA1 is that indoleacetic acid primarily affects cells with an activated pheromone response pathway. Interacting radial QTL would then arise if the underlying variants influence either the response to indoleacetic acid, or the activation of the pheromone response pathway by the BY allele of GPA1. The radial QTL include several loci involved in pheromone response, including the mating-type locus $M A T$, which dictates which pheromones are expressed or sensed (and is known to interact with $G P A I)^{30}$, and the gene VPS34, which is required for GPAl's activation of the mating pathway ${ }^{31}$. Further work will be required to elucidate the importance of the yeast pheromone response pathway for the fitness effects of indoleacetic acid.

For several other networks, the hub-QTL include candidate genes with known polymorphisms in BY and RM. For example, the networks regulating growth on media with Copper Sulfate and Manganese Sulfate have hub-QTL that includes the genes CUP1 and $P M R 1$, respectively. The BY allele of $C U P 1^{32}$ is known to increase copper ion tolerance ${ }^{33}$ and the RM allele of PMR1 allele confers manganese resistence ${ }^{34}$. The current data does not allow further functional dissection of the possible connections between these polymorphisms and the capacitation in the networks. However, by revealing the allelic dependencies between the loci in the network (Figure 3b; Figure 3d), it is possible to formulate hypotheses about how these and other known polymorphisms in hub-QTL could contribute epistatically to growth in the respective media for testing in future functional studies (Supplementary note).

In summary, we show that networks of capacitating genetic interactions are common, and that these networks form a key part of the genetic architectures of multiple complex traits in a large experimental yeast population. We illustrate how such interactions affect modelbased estimation of individual phenotypes and the inference of genetic architectures. This shows that epistasis needs to be explored beyond estimates of epistatic genetic variances, in order to understand its contribution to the phenotypic variability and long-term selection responses in populations. This is a key discovery in the long-standing debate about how to approach epistasis in complex trait research.

\section{URLs}

The R Project for Statistical Computing (accessed 17 January 2017), http://www.rproject.org; dglm: Double Generalized Linear Models. (accessed 17 January 2017), http:// cran.r-project.org/package $=\mathrm{dg} / \mathrm{m}$

\section{Online Methods}

The creation of the BYxRM cross, genotyping, phenotyping, quality control of genotypes, filtering and normalization of growth measurements has previously been described in $^{2,3}$. The reanalyzed data are available as supplementary information in ${ }^{3}$. Additive QTLs were 
mapped in Bloom et al. ${ }^{3}$. All analyses were performed using the R framework for statistical computing (http://www.r-project.org). All figures were prepared using R.

\section{Statistical analysis}

Inferring epistatic networks-Pairwise epistatic interactions were mapped by Bloom et $a l^{3}$. Networks of epistatic loci were inferred by connecting loci that displayed pairwise interactions. The R-package igraph ${ }^{35}$ was used to visualize individual networks and to identify network hubs. The GWA analysis for growth on indoleacetic acid containing medium among the segregants with the BY-allele at the hub-locus was performed using the qtscore function in the R-package Gen $A B E L^{36}$. Genome-wide significance was determined using a Bonferroni-corrected significance threshold for the number of tested markers

$\left(p<\frac{0.05}{28,220}=1.8 \times 10^{-6}\right)$. The additive genetic variance explained by a certain set of QTL was calculated as the $\mathrm{R}^{2}$ from a fixed effect model without interactions.

\section{Exhaustive mapping of loci in the network affecting growth on indoleacetic} acid containing medium-To identify all individual loci that contributed the additional genetic variance in amongst the segregants carrying the BY allele at the hub-QTL in the IAA-network, we performed a GWA analysis in this group of segregants using $G e n A B E L^{36}$ with significance thresholds as described above. This revealed in total 8 genome-wide significant loci in the radial network, out of which 6 were the same as the loci in the earlier two-way interaction analysis ${ }^{3}$.

Estimating average phenotypes for multi-locus genotypes-The average phenotypes were estimated for each of the $2^{6}=64$ possible combinations of alleles for 15 six-locus epistatic networks. Each of these networks had a hub-QTL connected to five radial loci by pairwise interactions. On average, $\frac{4,390}{64} \approx 69$ segregants are expected in each sixlocus genotype class in these networks, allowing confident estimation of the average growth associated with carrying each possible combination of alleles at these loci (exact values in each class are reported in the figures describing the results). Some of the hub-QTL were connected to more than five other loci in the network in the initial network analysis. Here we only kept the loci with the strongest statistical interaction with the hub-QTL as we could not confidently estimate phenotypes for individual genotype-classes in networks with more than six loci.

Estimation of the genetic variance heterogeneity at a locus-We estimated the difference in the phenotypic variance between segregants that carry alternative alleles at the epistatic loci using a Double Generalized Linear Model (DGLM) ${ }^{37}$, as suggested in Rönnegård et al. ${ }^{14}$. This allowed us to simultaneously model the effects of every locus on the phenotypic mean and variance. We fitted a DGLM with linear predictors for both mean and variance as $y \sim N\left(\mu_{1}+X \beta_{1}, e^{\iota 2}+X \beta_{2}\right)$ using the R-package $d g \operatorname{lm}$ (http://cran.rproject.org/package $=\operatorname{dg} 1 \mathrm{~m}$ ), where $y$ is the phenotype, $X$ is the genotype, $\beta_{1}$ is the effect on the mean, and $\beta_{2}$ is the effect on the variance. Coding the genotypes in $X$ as 0 and $1, \beta_{1}$ then describes the difference in mean whereas $e^{\beta^{2}}$ describes the fold difference in variance between the segregants with alternative alleles at the locus. 
Estimating the capacitating effects of the hub-QTL in the epistatic networksQTL interacting with 5 or more other loci were defined as hubs. We estimated the capacitating effects of all hub-QTL as follows. For each network containing a hub-QTL, we divided the segregants into two groups based on their genotype at the hub. We then fitted the mixed model $y=X \mu+a+e$ separately for each group. Here, $y$ is a column vector containing the phenotypes, $X$ is a column vector of ones, $\mu$ is the overall mean, $b \sim N\left(0, A \sigma_{a}^{2}\right)$ and $e \sim N\left(0, \sigma_{e}^{2}\right)$. $A$ is the additive kinship matrix, giving the fraction of the genome shared between each pair of segregants, $\sigma_{a}^{2}$ is the additive genetic variance captured by the markers, and $\sigma_{e}^{2}$ is the residual variance. $A$ was calculated using the $i b s$ function in the R-package GenABEL. We used the GenABEL function polygenic to fit the mixed model. The narrow sense heritability in each group was calculated as the intra class correlation $\rho=\frac{\sigma_{a}^{2}}{\sigma_{a}^{2}+\sigma_{e}^{2}}$.

We performed a permutation test to obtain the significance of the difference $\rho_{1}-\rho_{2}$ between the two groups of segregants in each network. For each of the 20 traits, we randomly divided the population into two groups and estimated $\rho_{1}$ and $\rho_{2}$ in these as described above. This was repeated 1000 times per trait to obtain 20 empirical NULL distributions. We here report the number of traits for which the difference $\rho_{1}-\rho_{2}$ was significant at a Bonferronicorrected multiple testing threshold of $\frac{0.05}{15} \approx 0.003$.

Quantifying non-linear effects after capacitation-To quantify the potential multiplicative action of capacitated radial alleles, we compared the fit of an additive ( $y=\mu+$ $x \beta+e)$ and an exponential $\left(y=\mu+\beta_{1}, e^{x \beta_{2}}+e\right)$ model. Here, $y$ is the phenotype, $x$ is the number of growth decreasing radial alleles, and $e$ is the residual variance.

\section{Modeling the phenotypes for individual multi-locus genotype classes-For}

each six-locus epistatic network, we estimated the phenotypes for the 64 individual genotype classes in each network using three different models including i) additive effects, ii) additive effects and pairwise interactions and iii) additive, pairwise and three-way interactions, respectively. For ii) and iii), the included interaction-terms that, together with all additive terms, minimized the $A I C=2 k-2 \ln (L)$. Here, $k$ is the number of included parameters and $L$ is the maximum likelihood value for the model. We used the R-function step in the stats package to perform the backward elimination (http://www.r-project.org). The performances of the final models (bias and accuracy) were evaluated using 10-fold cross-validation, where the variable selection for i) and ii) above was performed within the training data in every fold.

Within each of the 64 genotype-classes, defined by the six loci in each network, we calculated the CV estimation errors as $e=y-\hat{y}$. Here, $y$ is the actual and $\hat{y}$ the estimated phenotype. We tested if $e$ significantly deviated from 0 using a t-test. If the deviation was significant at a multiple testing Bonferroni corrected threshold of $\frac{0.05}{64} \approx 7.8 \times 10^{-4}$, we considered the estimate for that particular genotype-class biased. The accuracy of the estimates was measured by $e^{2}$, and for each genotype-class, we tested the difference in $e^{2}$ between models with and without interaction-terms using a t-test. If the $e^{2}$ was significantly 
lower for the interaction model, at a multiple testing Bonferroni corrected threshold of $\frac{0.05}{64} \approx 7.8 \times 10^{-4}$, we considered these estimates more accurate.

\section{Simulations}

In the simulations, we used the phenotypic means $\mu_{1} \cdots \mu_{64}$ in each of the $2^{6}=64$ classes for each of the 15 six-locus networks, and the total phenotypic variance $\sigma_{p}^{2}$ for each trait, obtained in the analyses above as a representation of the genetic architectures of these traits. In every simulation, we generated populations with the same number of segregants as in the original dataset $(n=4,390)$. The number of segregants in each genotype class was determined by the allele frequencies $p_{1} \cdots p_{6}$ at the six loci. For example, the number of observations with genotype $A B c D e f$, where the big/small letters indicate the alternative alleles at the six loci, would be $p_{1} \times p_{2} \times\left(1-p_{3}\right) \times p_{4} \times\left(1-p_{5}\right) \times\left(1-p_{6}\right) \times n$. To evaluate the effect of different combinations of allele-frequencies at the loci on the results, we simulated populations with $p_{\mathrm{k}} \in\{0.05,0.20,0.35,0.50,0.65,0.80,0.95\}$, where $k=1 \cdots 6$. This leads to, in total, $7^{6}$ allele frequency combinations. The phenotypes for the individuals in each genotype class were then simulated as $y_{k} \sim N\left(\mu_{k}, \sigma^{2}\right)$, where $k=1 \cdots 64$.

As a comparison, we also simulated populations where the genetic architectures (i.e. the phenotype for each of the 64 multi-locus genotypes) for the 15 networks were given by the estimates obtained from a six-locus additive model fitted to the respective loci. The linear model used was $\mathrm{y}=X \beta+e$, where $y$ is a column vector containing the phenotypes, $X$ is a $4,390 \times 7$ matrix, the first column consisting of ones and columns $2-7$ of the genotypes of the six loci, $\beta$ is a $1 \times 7$ column vector with the intercept and the additive genetic effects, and $e$ is the residual variance. Using this model, estimates $\widehat{\mu_{1}} \cdots \widehat{\mu_{64}}$ were obtained for each genotype class. The simulations were then performed across the different combinations of allele-frequencies as described above, with phenotypes given by $y_{k} \sim N\left(\widehat{\mu_{k}}, \sigma^{2}\right)$.

The additive genetic variance contributed by each locus was estimated as the $\mathrm{R}^{2}$ from the linear model $y=X \beta+e$, fitted to the respective subset of the $7^{6}$ simulated populations where the allele frequency of the locus itself was $0.5\left(p_{1}=0.5\right)$, and the frequency at the other five loci varied in $p_{\mathrm{k}} \in\{0.05,0.20,0.35,0.50,0.65,0.80,0.95\}$, where $k=2 \cdots 6$. In this model, $X$ is a column vector with the genotype of the locus whose allele frequency is 0.5 . The additive genetic variances were estimated in such subsets to analyze the effect of the individual locus across variable genetic backgrounds for the other loci, without the estimates being influenced by changes to the allele frequency of the analyzed locus itself.

\section{Code Availability Statement}

The custom R-code for the analyses and simulations in the manuscript has been deposited in Github, where it is available at https://github.com/simfor/yeast-epistasis-paper.

\section{Supplementary Material}

Refer to Web version on PubMed Central for supplementary material. 


\section{Acknowledgments}

Funding was provided by the Howard Hughes Medical Institute and NIH grant R01 GM102308 (L.K.), F32 GM116318 (M. J. S.) and Swedish Research Council grant 621-2012-4632 (Ö.C.).

\section{References}

1. Hill WG, Goddard ME, Visscher PM. Data and theory point to mainly additive genetic variance for complex traits. PLoS Genet. 2008; 4:e1000008. [PubMed: 18454194]

2. Bloom JS, Ehrenreich IM, Loo WT, Lite TLV, Kruglyak L. Finding the sources of missing heritability in a yeast cross. Nature. 2013; 494:234-7. [PubMed: 23376951]

3. Bloom JS, et al. Genetic interactions contribute less than additive effects to quantitative trait variation in yeast. Nat Commun. 2015; 6:8712. [PubMed: 26537231]

4. Polderman TJC, et al. Meta-analysis of the heritability of human traits based on fifty years of twin studies. Nat Genet. 2015; 47:702-9. [PubMed: 25985137]

5. Cheverud JM, Routman EJ. Epistasis and its contribution to genetic variance components. Genetics. 1995; 139:1455-61. [PubMed: 7768453]

6. Carlborg O, Haley CS. Epistasis: too often neglected in complex trait studies? Nat Rev Genet. 2004; 5:618-625. [PubMed: 15266344]

7. Mackay TFC. Epistasis and quantitative traits: using model organisms to study gene-gene interactions. Nat Rev Genet. 2014; 15:22-33. [PubMed: 24296533]

8. Nelson RM, Pettersson ME, Carlborg Ö. A century after Fisher: time for a new paradigm in quantitative genetics. Trends Genet. 2013; 29:669-76. [PubMed: 24161664]

9. Gerke J, Lorenz K, Cohen B. Genetic interactions between transcription factors cause natural variation in yeast. Science. 2009; 323:498-501. [PubMed: 19164747]

10. Le Rouzic A, Siegel PB, Carlborg O. Phenotypic evolution from genetic polymorphisms in a radial network architecture. BMC Biol. 2007; 5:50. [PubMed: 18001473]

11. Le Rouzic A, Carlborg Ö. Evolutionary potential of hidden genetic variation. Trends Ecol Evol. 2008; 23:33-37. [PubMed: 18079017]

12. Carlborg O, Jacobsson L, Ahgren P, Siegel P, Andersson L. Epistasis and the release of genetic variation during long-term selection. Nat Genet. 2006; 38:418-20. [PubMed: 16532011]

13. Paixão T, Barton NH. The effect of gene interactions on the long-term response to selection. Proc Natl Acad Sci U S A. 2016; 113:4422-4427. [PubMed: 27044080]

14. Rönnegård L, Valdar W. Detecting major genetic loci controlling phenotypic variability in experimental crosses. Genetics. 2011; 188:435-47. [PubMed: 21467569]

15. Rönnegård L, Valdar W. Recent developments in statistical methods for detecting genetic loci affecting phenotypic variability. BMC Genet. 2012; 13:63. [PubMed: 22827487]

16. Rutherford SL, Lindquist S. Hsp90 as a capacitor for morphological evolution. Nature. 1998; 396:336-342. [PubMed: 9845070]

17. True HL, Lindquist SL. A yeast prion provides a mechanism for genetic variation and phenotypic diversity. Nature. 2000; 407:477-483. [PubMed: 11028992]

18. Gibson G, Dworkin I. Uncovering cryptic genetic variation. Nat Rev Genet. 2004; 5:681-690. [PubMed: 15372091]

19. Paaby AB, Rockman MV. Cryptic genetic variation: evolution's hidden substrate. Nat Rev Genet. 2014; 15:247-58. [PubMed: 24614309]

20. Falconer, DS., Mackay, TFC. Introduction to quantitative genetics. 4. Longmans Green; Harlow, Essex, UK: 1996.

21. Bateson W. Facts Limiting the Theory of Heredity. Science. 1907; 26:649-660. [PubMed: 17796786]

22. Monnahan PJ, Kelly JK. Epistasis Is a Major Determinant of the Additive Genetic Variance in Mimulus guttatus. PLOS Genet. 2015; 11:e1005201. [PubMed: 25946702]

23. Bergman A, Siegal ML. Evolutionary capacitance as a general feature of complex gene networks. Nature. 2003; 424:549-552. [PubMed: 12891357] 
24. Masel J, Siegal ML. Robustness: mechanisms and consequences. Trends Genet. 2009; 25:395-403. [PubMed: 19717203]

25. Queitsch C, Sangster Ta, Lindquist S. Hsp90 as a capacitor of phenotypic variation. Nature. 2002; 417:618-24. [PubMed: 12050657]

26. Dworkin I, Palsson A, Birdsall K, Gibson G. Evidence that Egfr Contributes to Cryptic Genetic Variation for Photoreceptor Determination in Natural Populations of Drosophila melanogaster. Curr Biol. 2003; 13:1888-1893. [PubMed: 14588245]

27. Lachowiec J, Shen X, Queitsch C, Carlborg Ö. A Genome-Wide Association Analysis Reveals Epistatic Cancellation of Additive Genetic Variance for Root Length in Arabidopsis thaliana. PLoS Genet. 2015; 11:e1005541. [PubMed: 26397943]

28. Yvert G, et al. Trans-acting regulatory variation in Saccharomyces cerevisiae and the role of transcription factors. Nat Genet. 2003; 35:57-64.

29. Lang GI, Murray AW, Botstein D. The cost of gene expression underlies a fitness trade-off in yeast. Proc Natl Acad Sci U S A. 2009; 106:5755-60. [PubMed: 19299502]

30. Brem RB, Storey JD, Whittle J, Kruglyak L. Genetic interactions between polymorphisms that affect gene expression in yeast. Nature. 2005; 436:701-703. [PubMed: 16079846]

31. Slessareva JE, Routt SM, Temple B, Bankaitis VA, Dohlman HG. Activation of the phosphatidylinositol 3-kinase Vps34 by a G Protein alpha subunit at the endosome. Cell. 2006; 126:191-203. [PubMed: 16839886]

32. Fogel S, Welch JW, Maloney DH. The molecular genetics of copper resistance in Saccharomyces cerevisiae — a paradigm for non-conventional yeasts. J Basic Microbiol. 1988; 28:147-160. [PubMed: 3057171]

33. Perlstein EO, Ruderfer DM, Roberts DC, Schreiber SL, Kruglyak L. Genetic basis of individual differences in the response to small-molecule drugs in yeast. Nat Genet. 2007; 39:496-502. [PubMed: 17334364]

34. Sadhu MJ, Bloom JS, Day L, Kruglyak L. Mapping Without Crosses. Science. 2016; 352:1113-6. [PubMed: 27230379]

35. Csárdi G, Nepusz T. The igraph software package for complex network research. InterJournal Complex Syst. 2006; 1695:1695.

36. Aulchenko YS, Ripke S, Isaacs A, van Duijn CM. GenABEL: an R library for genome-wide association analysis. Bioinformatics. 2007; 23:1294-6. [PubMed: 17384015]

37. Smyth GK. Generalized Linear Models with Varying Dispersion. Journal of the Royal Statistical Society B. 1989; 51:47-60. 
a

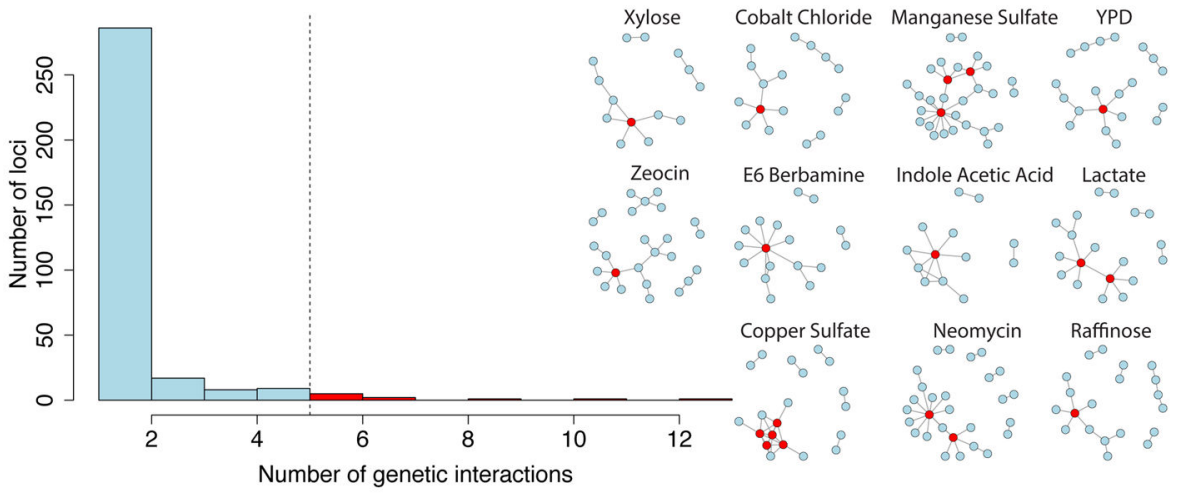

b networks

a) A histogram of the number of interactions each epistatic QTL is involved in. Most of the 330 epistatic loci detected for the 20 traits are involved in few pairwise interactions. Few QTL are involved in many interactions, here defined as five or more, but their role is prominent since they are the hubs that tie the networks together (b). b) The pairwise QTL interactions contributing to growth in 11 different media form highly interconnected epistatic networks. Each circle represents a QTL and the lines represent significant pairwise interactions ${ }^{3}$. Red circles highlight hub-QTL involved in five or more pairwise interactions. 
a

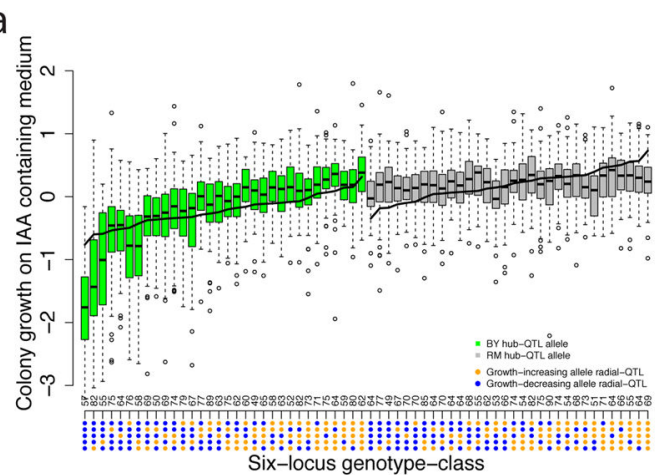

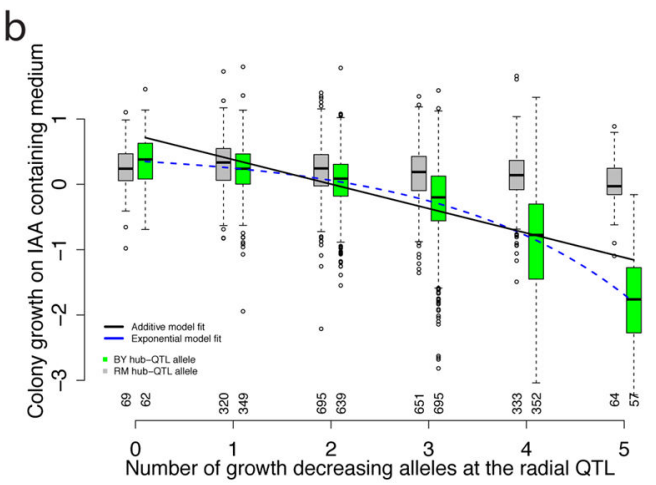

Figure 2. Epistatic network regulating growth on indoleacetic acid containing medium The RM allele suppresses (canalizes) the phenotypic effects of segregating alleles at the radial QTL. The BY allele capacitates the growth decreasing effects that combine in a nonlinear fashion. Tukey boxplots (bottom/top of box are first/third quartiles, band is median and ends of whiskers the lowest/highest datum within 1.5 interquartile range of the lower/ upper quartile) illustrate the phenotypic distributions in segregants with different combinations of alleles across the IAA-network. a) One boxplot for each of the 64 genotype classes. The color indicates the genotype at the hub-QTL (chrVIII:114,114bp; Green/grey boxes for BY/RM alleles). The $\mathrm{x}$-axis gives the six-locus genotype class, where blue/orange dots indicates growth-decreasing/increasing alleles at the five radial-QTL (from top to bottom chrXIV:469,224bp, chrIII:198,615bp, chrIV:998,628bp, chrXIII:410,320bp, and chrXII:645,539bp). The black line through the boxes illustrates the additive model based estimates of the phenotypes for the 64 genotype classes. The number above the $\mathrm{x}$-axis is the number of segregants in each genotype class. b) One boxplot for each group of segregants that share the same number of growth decreasing alleles at the five radial QTL. The segregants are divided and colored based on the genotype at the hub-QTL as in a). The Xaxis gives the number of growth decreasing alleles at the radial QTL and the number of segregants in each group. The regression lines illustrate the fit for linear additive (black; R2 $=0.34$ ) and non-linear exponential (dashed blue; R2 =0.39) models, respectively. 

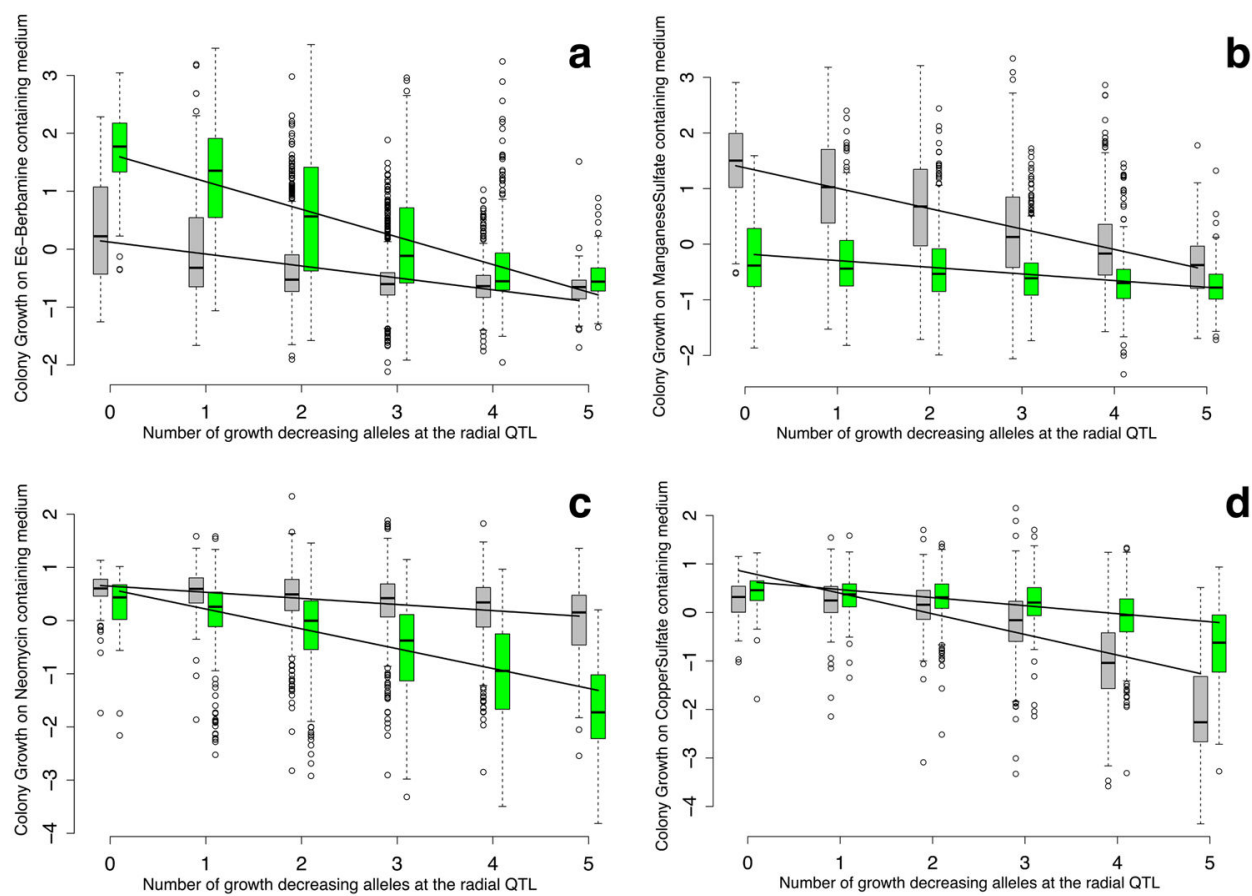

Figure 3. Epistatic networks contain hub-QTL capacitor alleles of both BY and RM origin that moderate growth increasing or decreasing effects of segregating alleles at radial QTL

Each sub-figure corresponds to one epistatic network where the hub-QTL capacitates the growth-increasing, or growth-decreasing, effects of segregating alleles at the radial QTL (a: E6-Berbamine, b: Manganese Sulfate, c: Neomycin, d: Copper Sulfate). Within each network, the segregants are divided based on the genotype at the hub-QTL, and segregants carrying the RM allele are shown in grey and those with the BY allele are shown in green. The $\mathrm{X}$-axis gives the number of growth decreasing alleles at the radial QTL. The Tukey boxplots (bottom/top of box are first/third quartiles, band is median and ends of whiskers the lowest/highest datum within 1.5 interquartile range of the lower/upper quartile) show phenotypic distributions for groups of segregants with different numbers of growth affecting alleles at the five radial QTL in six-locus epistatic networks. a(b) illustrate networks where the RM(BY) allele at the hub-QTL maintain low growth in the segregants almost regardless of the genotypes at the radial-QTL. In $\mathbf{c}(\mathbf{d})$, the $\mathrm{RM}(\mathrm{BY})$ alleles allow the segregants to maintain high growth almost regardless of which alleles segregate at the radial QTL. The regression lines illustrate the difference in effects of the radial-QTL alleles depending on the genotype at the hub-QTL. 


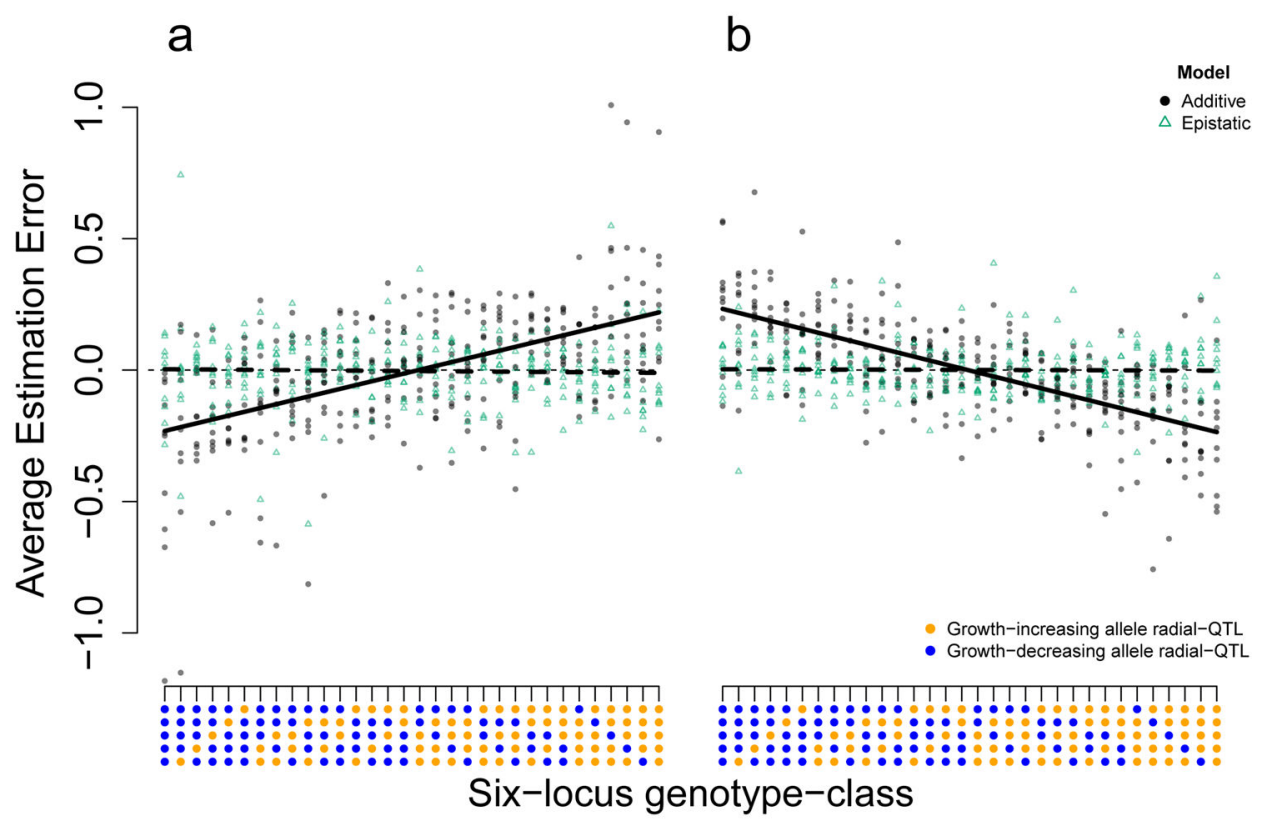

Figure 4. The biases in the additive model based estimates of the phenotypes are largest in the genotype-classes with the largest or poorest expected growth

Across the 10 interaction networks with a capacitor hub-QTL, the most extreme additive model based representations of individual multi-locus genotypes are biased. The additive model under/over-estimates the extremity of the genotypes with many/few growthdecreasing alleles at the radial-QTL. This results in a positive correlation between the estimation errors (bias) and the number of growth-increasing alleles at the radial-QTL when there is a capacitor allele at the hub-QTL $(\mathbf{a} ; \mathrm{r}=0.54$; Pearson correlation; $\mathrm{n}=320 ; p<2.2 \times$ $10^{-16}$ in two-sided test). Among segregants with a non-capacitor allele at the hub-QTL (b) the trend is opposite with over/under- estimates for segregants with many/few growthdecreasing alleles at the radial-QTL $\left(\mathrm{r}=-0.69\right.$; Pearson correlation; $\mathrm{n}=320 ; p<2.2 \times 10^{-16}$ in two-sided test). The $y$-axis gives the average estimation-error (bias) from cross-validation for each genotype in the 10 networks. The $\mathrm{x}$-axis illustrates the genotype at the five radial QTL in the networks: blue/orange dots indicate growth-increasing/decreasing alleles, respectively. Each dot represents the average estimation error (bias) for a particular six-locus genotype-class in one of the 10 networks. Black dots (green triangles) are errors from additive-only (additive + pairwise interaction) models with solid (dashed) black lines denoting the regression on these errors. The estimation errors (biases) are calculated as measured - estimated phenotypes, and over/under estimations will therefore result in negative/positive estimation errors (biases) respectively. 


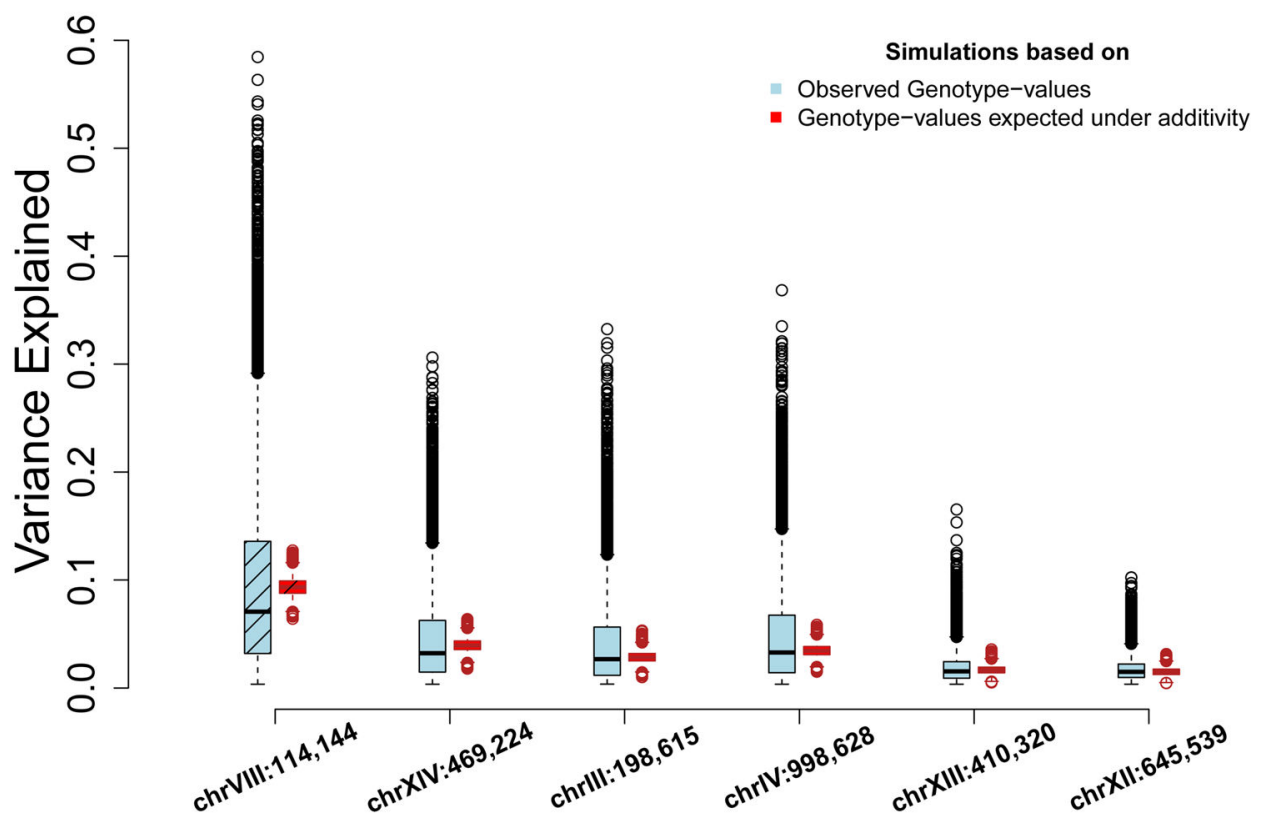

Figure 5. Simulations show that the additive genetic variances contributed by the loci in the epistatic network regulating growth on indoleacetic acid containing medium is highly dependent on the allele-frequencies at the other loci in the same network

The phenotypic variance explained by the individual loci in the IAA-network depends on the allele frequencies at the other loci with which they interact. Each Tukey boxplot (bottom/top of box are first/third quartiles, band is median and ends of whiskers the lowest/highest datum within 1.5 interquartile range of the lower/upper quartile) shows the additive genetic variances across 16,807 simulated populations. For each locus, we simulated populations where the allele frequency of the loci themselves were fixed at 0.5 , while varying the frequencies at the other 5 loci from 0.05 to 0.95 in increments of 0.15 . The light blue boxes represent the variability in the additive genetic variances when simulating populations based on the observed phenotypic means for the 64 genotype classes in the IAA-network. The red boxes represent the additive genetic variances when simulating based on the phenotypic means estimated using the additive genetic model (black line in Figure 2a). The $x$-axis gives the location of the SNP representing each locus (chromosome:location in bp). The two leftmost boxplots (diagonal lines) show the simulation results for the hub-locus. 